\title{
Exploraciones sobre las concepciones y prácticas del cuerpo y la comunicación no verbal: la escuela, el proceso de aprendizaje y el aula de lengua extranjera
}

Quiñones, Jesús Antonio; Romero Melo, Jenny Esperanza

Exploraciones sobre las concepciones y prácticas del cuerpo y la comunicación no verbal: la escuela, el proceso de aprendizaje y el aula de lengua extranjera

Revista Educación, vol. 44, núm. 2, 2020

Universidad de Costa Rica, Costa Rica

Disponible en: http://www.redalyc.org/articulo.oa?id=44062184022

DOI: https://doi.org/10.15517/revedu.v44i2.38945

Esta obra está bajo una Licencia Creative Commons Atribución-NoComercial-SinDerivar 3.0 Internacional. 


\section{Exploraciones sobre las concepciones y prácticas del cuerpo y la comunicación no verbal: la escuela, el proceso de aprendizaje y el aula de lengua extranjera}

Explorations about the Conceptualizations and Practices about the Body and the Non- verbal Communication: The School, the Learning Process and the Foreign Language Classroom.

Jesús Antonio Quiñones

Universidad de las Américas y el Caribe, México

DOI: https://doi.org/10.15517/revedu.v44i2.38945

dr_jesus_quinones@funcea.unac.edu.mx

(iD http://orcid.org/0000-0002-7725-504X

Jenny Esperanza Romero Melo

Universidad de la Sabana, Colombia

jennyrome@unisabana.edu.co

(iD) http://orcid.org/0000-0002-7687-8667

Recepción: 04 Octubre 2019

Aprobación: 13 Mayo 2020

\section{ReSUMEN:}

El presente estudio tuvo como propósito describir los modos en los cuales se aborda la comunicación no verbal en el aula de EFL, asimismo, sus relaciones posibles con las concepciones y prácticas sobre el cuerpo en el contexto de un colegio femenino en Bogotá, Colombia. Los sujetos elegidos consistieron en un grupo de 42 estudiantes de grado décimo entre los 14 y 17 años, quienes manifestaron dificultades, incomodidad y temor en el uso de la comunicación no verbal en el marco de la clase de lengua extranjera. Esta investigación se fundamenta en el paradigma cualitativo a partir del cual se efectuó un estudio de caso en las siguientes fases: diseño de preguntas de reflexión, recopilación de datos y el análisis e interpretación de la información y hallazgos. Asimismo, se hizo uso de instrumentos como la observación, el cuestionario y la entrevista semiestructurada. Como resultado se encuentra que el cuerpo en la escuela se configura desde una perspectiva física, biológica y moral. Al tiempo que las estudiantes consideran que el cuerpo no es relevante en su aprendizaje, se evidencia falta de educación sobre este en sus dimensiones experiencial, comunicativa, simbólica y cultural. De ahí que este texto busque replantear la importancia del cuerpo en el contexto educativo, lo cual implicaría incluir al cuerpo en proceso interdisciplinar que desarrolle la construcción de subjetividad y las posibilidades de interacción con la otredad, entendida desde las particularidades de la cultura extranjera.

Palabras clave: Cuerpo, Comunicación no verbal, Cuerpo como comunicador, Comunicación no verbal en el aprendizaje de idiomas, Aprendizaje de idiomas.

\section{Abstract:}

This study describes how to address nonverbal communication in EFL classrooms and how it may be related to concepts and practices regarding the body within the context of an all-girls school in Bogotá, Colombia. The subjects of the study are 42 tenth-grade female students ranging from 14 to 17 years of age, who were experiencing difficulty and fear in using nonverbal communication in their foreign language class. This is a qualitative study that was conducted in various phases based on a descriptive case study that involved: design of thought-provoking questions, data collection, data analysis and interpretation, and findings. The methodology was based on observation, questionnaires and semi-structured interviews. Results reveal that, at school, the human body is configured according to physical, biological and moral perspectives. At the same time, students state that they do not consider the body relevant to learning. This reflects an overall lack of knowledge about the experiential, communicative, symbolic and cultural dimensions of the body. This study emphasizes the importance of the body within a school context that should be included as part of an interdisciplinary process to support the construction of subjectivity and the possibility of interacting with one's otherness which, in this case, is understood as the particularities of a foreign culture.

KEYWORDS: Body, Nonverbal Communication, Body Language, Nonverbal Communication in Language Learning, Language Learning. 


\section{INTRODUCCIÓN}

A pesar de las transformaciones que se han efectuado en las prácticas pedagógicas y didácticas en la escuela, la educación del cuerpo sigue efectuándose en términos disciplinares y regulatorios que lo desconocen como comunicador. Uno de los factores más decisivos en este fenómeno ha sido la desarticulación entre saberes como la lecto-escritura, las ciencias naturales y sociales, las matemáticas y el cuerpo. Como lo indica Scharagrodsky (2007), en el contexto educativo, existe "un cuerpo sin voz ni voto" (p.3). Esto implica que la importancia del cuerpo en la construcción de conocimiento no ha sido considerada, el cuerpo se entiende como un organismo biológico y funcional, sin embargo, sus posibilidades simbólicas, expresivas y comunicativas no han tenido cabida en la estructuración del currículo. Como resultado, las relaciones entre el saber disciplinar, el cuerpo y la comunicación no verbal son desatendidas en la escuela y por tanto inexploradas en su efecto sobre la construcción subjetiva del estudiantado (Gómez, 2009). En efecto, a través del contexto elegido para el presente estudio, se evidencia que las jóvenes demuestran temor, apatía, incluso resistencia durante las prácticas corporales y no verbales durante la clase de lengua extranjera. De ahí que, este estudio de caso tenga como propósito analizar e interpretar las posibles relaciones entre las concepciones y prácticas sobre el cuerpo en la escuela y cómo estas podrían tener un impacto sobre el aprendizaje de la lengua extranjera.

\section{JuSTIFICACIÓN}

El cuerpo es imprescindible en el proceso de aprendizaje; sin embargo, las concepciones dualistas que lo separan de la mente y por tanto del pensamiento siguen imperando en la escuela (Arévalo, 2018). Muestra de ello es la carencia de una educación sobre el cuerpo que no solo lo comprenda como materialidad física. De modo que el cuerpo se aborde como experiencia subjetiva y a su vez como comunicador. Por consiguiente, es necesario dar cuenta de las concepciones y prácticas del cuerpo en la escuela: las definiciones de estudiantes, las normatividades que lo regulan y su integración en disciplinas distintas a la educación física, entre otros aspectos. En particular, este artículo pretende indagar acerca de la mirada que se da a la concepción de cuerpo mientras que tiene lugar el proceso de enseñanza-aprendizaje de lengua extranjera.

La observación sobre el entorno de aprendizaje de este estudio ratifica que la educación sobre el cuerpo en el país es reducida. Como lo señalan Cabra y Escobar (2014):

No se encuentran investigaciones que se pregunten por el lugar que ocupa el cuerpo en la clase de matemáticas, biología o español, confirmando la idea de que este solo existe en la escuela cuando se trata de los pocos espacios que se dedican a trabajar sobre el movimiento (p.149)

Así entonces, el abordaje del cuerpo en la escuela y las concepciones que tiene el estudiantado sobre este manifiestan la necesidad de repensar su lugar en el proceso de aprendizaje. El presente estudio busca explorar el lugar del cuerpo en la clase de lengua extranjera. En particular, dar cuenta de las relaciones y prácticas que las estudiantes construyen entre el aprendizaje lingüístico y el cuerpo en la comunicación. Así entonces, argumentar la importancia de construir interdisciplinaridad sobre el cuerpo y la comunicación no verbal como parte de la construcción subjetiva y de aprendizaje del educando.

En esta medida:

el cuerpo no solo es considerado como el fenómeno más completo y valioso a la hora de comprender hechos tales como las ópticas de la vida, la voluntad de creación de máscaras y metáforas, etc., sino también como el fenómeno de más acervo probatorio cuando se trata de ser fiel a la vida (Rocha, 2000, p.164)

La experiencia vital y su comprensión se dan a partir del cuerpo, por tanto, el acto de aprender solo es posible a través de este. Es allí donde se manifiesta el lenguaje como entramado lingüístico, gestual, kinésico, 
espacial, interaccional y artístico. Se requiere una exploración de estas posibilidades de aprendizaje y esto implica una escuela que no solo entienda al cuerpo como un hecho físico indicador de comportamiento sino una escuela que permita el hecho creativo en el cuerpo como simbólico y comunicador.

En este orden de ideas, el cuerpo es concebido como texto. En el cual se elabora desde diversos elementos que le permiten al sujeto expresarse y entrar en relación con el mundo. Dicho cuerpo contempla la no verbalidad como elemento comunicativo. A saber, el cuerpo desde la CNV (Comunicación no verbal) incluye: gestos, miradas, kinésica, proxémica, señas, vestuario. Por supuesto, estos elementos a través de la interacción son ejercidos congruentes con la cultura. Los códigos no verbales y sus aspectos expresivos si bien tienen un grado de universalidad son matizados según las particularidades de ciertas formas de vida. En consecuencia, el conocimiento de estos códigos conduce a un reconocimiento y acercamiento al otro, a la diferencia.

Con esta resignificación del cuerpo en la escuela, se identifica la necesidad de hacer vivida la lengua extranjera en el aula. Puesto que:

Es obvio que una enseñanza de L2 centrada solo en los elementos verbales traerá consigo una comunicación artificial limitada al contexto del aula, e incompleta ya que los estudiantes no podrán cooperar de manera activa en el proceso comunicativo ni como emisores ni como receptores. (Álvarez, 2002, p.9).

A partir de este estudio se expone esta complejidad. En el aula de lengua extranjera son comunes los ejercicios que pretenden la puesta en práctica de las habilidades de producción. Sin embargo, no son pocas las veces en que tales ejercicios obedecen a una necesidad académica marcada por la memorización. No hay entonces una aprehensión del aprendizaje de lengua extranjera en sus constitutivos expresivos, comunicativos, expresivos y de encuentro con la diferencia.

En resumen, con este trabajo se pretende explorar en primera medida el abordaje del cuerpo en la escuela y las relaciones que la población establece entre el cuerpo, el aprendizaje y la comunicación. De esta forma visibilizar la importancia de considerar el cuerpo desde lo fenomenológico y simbólico, lo cual conlleva a entenderlo desde sus elementos comunicativos y expresivos. El cuerpo como lugar de encuentro con una cultura distinta, articulador de aspectos verbales y no verbales que le permita al estudiantado un acercamiento auténtico a la lengua extranjera.

\section{AnTECEDEnTES}

\section{La escuela y el cuerpo}

La adecuación del cuerpo: prácticas aceptadas y no aceptadas en contexto escolar.

En el texto titulado El cuerpo en la Escuela de Scharagrodsky (2007), publicado por el Ministerio de Educación, Ciencia y Tecnología de Argentina, se dan a conocer las prácticas sobre el cuerpo en el contexto escolar. Así pues, se efectúa una exploración del cuerpo en la modernidad, la escolarización de los cuerpos, los cuerpos femeninos y masculinos, la corporalidad actual en la escuela, los mercados, consumos corporales y estéticas juveniles en la escuela. Se argumenta que las disposiciones disciplinares, homogenizantes y binarias con respecto al cuerpo se han ido debilitando en la escuela. Sin embargo, se evidencian contradicciones que dan paso a la desigualdad de los cuerpos la cual no se considera en el escenario educativo. Esta investigación resulta ser relevante para este estudio en cuanto expone la historicidad del cuerpo al interior de la escuela, sin embargo, se distancia de lo observado en el escenario educativo seleccionado, pues las categorías binarias entre lo femenino y lo masculino siguen estando vigentes y teniendo un impacto sobre las perspectivas que las jóvenes tienen sobre el papel del cuerpo y la normativa institucional.

De manera similar, en el artículo Saber, Cuerpo y Escuela: el uso de los sentidos y la educación somática de Pedraza (2010), se articula el saber, el poder y el cuerpo a partir de las prácticas en la escuela. El texto toma elementos como las pedagogías del conocimiento, las comprensiones del cuerpo, los modelos educativos, el 
vínculo social de la relación escuela, así como el conocimiento y el ordenamiento del cuerpo. Desde estas conceptualizaciones, señala la separación entre los saberes abstractos y el cuerpo en la escuela, de ahí que, proponga una unificación de ambos en el proceso de aprendizaje. Si bien el texto da cuenta de la historicidad en relación con el cuerpo, el saber y el contexto educativo de la cual se vale para efectuar una plantear un currículo unificador, no señala procedimientos concretos para llevar esto a cabo. Dicho proceso debe partir de una transformación epistémica sobre la concepción del cuerpo, como lo expondrán los resultados del presente estudio.

Entretanto, en la investigación Efectos del disciplinamiento en la escuela en la configuración de subjetividad de Chavarriaga y Ochoa (2015), se abordan las relaciones de poder en el ejercicio y resistencia de las normatividades institucionales. A través de la genealogía planteada por Foucault, se toma como referencia una institución oficial de Medellín. Como parte de los resultados, se expone la necesidad de hacer una lectura distinta del cuerpo en el ámbito escolar, puesto que se le otorga un lugar primordial a lo cognitivo y racional sobre otras posibilidades de aprender. Asimismo, se reconoce que la subjetividad representada en el estudiantado no reconoce el valor de las normas, ya que no participa en su construcción y están desfasadas de sus intereses. La pertinencia de este trabajo recae en que señala cómo las concepciones sobre el cuerpo están mediadas por la regulación de su comportamiento y visiones racionales que no consideran las subjetividades de los educandos. No obstante, el texto podría haber explorado cómo las prácticas disciplinares sobre el cuerpo interfieren sobre el proceso académico y el saber disciplinar; así pues, el presente estudio pretende dar cuenta de dichas relaciones.

\section{El cuerpo y su invisibilidad en el proceso académico}

La investigación El cuerpo en la escuela: subjetividades de jóvenes de las Instituciones Educativas Distritales Alemania Unificada y Fabio Lozano Simonelli de la ciudad de Bogotá de Niño (2015), plantea describir las subjetividades que se constituyen como cuerpos-sujetos. Para ello se utilizó la metodología hermenéutica con enfoque etnográfico. Como conclusiones se tiene que el cuerpo es un escenario epistémico, ético y político; asimismo, ejerce la resistencia desde la creatividad frente a la norma escolar. Al igual que los trabajos anteriores, este estudio enfatiza en la necesidad de reconfigurar las concepciones sobre el cuerpo considerándolo como parte esencial de la construcción subjetiva y simbólica, sin embargo, deja de lado las implicaciones de la invisibilización del cuerpo en el saber disciplinar y cómo la resistencia de los jóvenes frente a la norma da cuenta de un desfase entre sus perspectivas y aquellas de orden institucional.

Asimismo, el artículo Reflexiones acerca de la corporeidad en la escuela: hacia la despedagogización del cuerpo de Pateti (2007) consiste en la reflexión de una investigación cualitativa interpretativa y análisis contextual de opiniones y remembranzas de estudiantes de educación y del profesorado de la Universidad Pedagógica Experimental Libertador. De este trabajo se indica la importancia de vincular todo acto educativo a la corporalidad, dado que se articula con lo intelectual para en el proceso de aprendizaje. Esto en vista que, en palabras de la autora, la escuela continúa desestimando la actividad motriz - el cuerpo - para la adquisición de saberes. Este estudio manifiesta la necesidad de articular el cuerpo con el proceso de aprendizaje, en línea con lo expresado por Pedraza (2010), partiendo de un contexto educativo distinto basado en la educación experimental. A diferencia de otros trabajos, no se ahonda sobre cómo las concepciones del cuerpo mediatizan esta articulación, de igual modo, podría presentarse un análisis más cuidadoso sobre las implicaciones de la despedagogización del cuerpo.

De otra parte, el libro El cuerpo en Colombia: Estado del arte cuerpo y subjetividad de Cabra y Escobar (2014) da a conocer una serie de reflexiones sobre el cuerpo en el país de orden social, histórico, teórico. El apartado El cuerpo y la escuela: subjetividades en tensión se exponen reflexiones sobre la relación cuerpoescuela, el movimiento, el cuerpo, la historia y la educación. Algunas de las conclusiones consisten en: la concepción de la escuela como un panóptico que regula el cuerpo y la falta de conceptualización del cuerpo 
en la educación del país. En particular, se resalta que la reflexión sobre el cuerpo compete a la motricidad y queda excluida de los procesos de aprendizaje intelectuales.

\section{Cuerpo simbólico y comunicación no verbal}

En el artículo El aprendizaje simbólico del cuerpo de Bárcena y Melich (2000), se plantea la cuestión del sentido como inherente a la experiencia humana y por tanto, al cuerpo. Así entonces, el cuerpo, además de su materialidad, tiene una dimensión simbólica que involucra que el cuerpo-texto sea leído e interpretado por otros. No obstante, en esta experiencia sobre el cuerpo, la pedagogía juega un rol restrictivo, nominativo y vigilante; según los autores, Elpoder pedagógico califica, mide, aprecia, jerarquiza. La aportación fundamental de este trabajo consiste en explicitar cómo el cuerpo construye y comunica significados, a pesar de esto, la escuela se aproxima al cuerpo desde una perspectiva evaluativa y disciplinar. Al igual que el anterior estudio se hace hincapié sobre el poder pedagógico sin embargo, podría precisarse desde qué enfoque se define la pedagogía sobre sobre el cuerpo y si esta equivale a la normativa institucional sobre este. Como resultado, el presente estudio no parte de la pedagogía sobre el cuerpo sino en concreto, normativas institucionales; puesto que, hay consideraciones de orden epistémico y de enfoque educativo de mayor envergadura al hablar de pedagogía(s) del cuerpo.

De manera similar, en el artículo El cuerpo en la educación da quépensar: perspectivas hacia una educación corporal de Gallo (2009), se insiste en una conceptualización fenomenológica del cuerpo. Dicha concepción involucra repensar el cuerpo en términos de lo físico a través de los cuales este se objetiviza, de ahí que, el cuerpo se entienda desde sus inagotables capacidades significativas. Ahora, el papel de la pedagogía, según el texto es situar al sujeto en relación con el mundo y esto conduce a considerar el cuerpo en su discursividad. Esta posibilidad es elaborada a partir del concepto quiasmo de Merleau Ponty en el cual desde un orden fenomenológico se busca superar conceptos duales, es decir, desde singularidad del ser más que como el cuerpo-mente. Este estudio, a diferencia de los referidos, da respuesta a lo que significaría la pedagogía del cuerpo desde un enfoque discursivo y postmoderno. No obstante, deja de lado relaciones históricas y conceptuales entre el saber disciplinar y el cuerpo que se plantearían como un primer obstáculo para superar conceptualizaciones duales sobre este; es decir, que no puede evidenciarse una hoja de ruta desde el currículo que permita el ejercicio de estos nuevos postulados teóricos sobre el cuerpo.

\section{La comunicación no verbal en el proceso de aprendizaje}

En el artículo Non verbal communication: An integral part of teaching learning process de Bunglowala (2015), se argumenta que la comunicación no verbal no es solo una extensión de lo lingüístico sino en sí misma es fundamental en el proceso comunicativo. Así pues, en el aprendizaje se comprueba que la CNV (Comunicación no Verbal) mejora la comprensión e incrementa el interés del alumnado frente al tema. Además, la utilización de expresiones faciales, gestos, postura, contacto visual y proxémica influye emocionalmente en la relación entre el personal docente y el cuerpo estudiantil. La contribución del estudio radica en hacer énfasis en la necesidad de educar sobre la comunicación no verbal de manera distinta al saber lingüístico, sin embargo, el plantear la CNV como integral en el proceso de aprendizaje conllevaría explorar las perspectivas del estudiantado frente este y cómo efectuar procesos metacognitivos en el que el educando comprenda la importancia de la CNV en su proceso académico. Este estudio busca descubrir estas perspectivas y cómo permitirían o limitarían la enseñanza de la CNV.

Entretanto, el artículo Use of Nonverbal Communication in the Classroom as a Way of Enhancing Classroom Teaching: A Case Study of Solusi High School, Zimbabwe de Muchemwa (2013) se basa en un estudio de caso sobre la CNV en la enseñanza en el aula. Consiste en una descripción del uso de la CNV por parte 
del personal docente en su práctica instructiva y disciplinaria. Dicha investigación combina la observación y la entrevista como instrumentos de recolección de datos. De acuerdo con los hallazgos, en contraste con el estudio anterior, el profesorado usa de modo sofisticado técnicas en su CNV según su experiencia. Si bien el estudio indica la importancia de la CNV en la enseñanza, se parcializa desde el lado del cuerpo docente y cómo el uso de la CNV puede potenciar la mediación de saberes con el alumnado; es decir, no se efectúa una exploración del uso de la CNV por parte del cuerpo estudiantil, quien en últimas es el constructor de su propio saber, ni tampoco cómo su no verbalidad canaliza la interacción con el cuerpo docente.

Por su parte en el trabajo Evaluación de la comunicación no verbal en los estudiantes de $9^{\circ}$ en la clase de lengua castellana para mejorar sus prácticas expositivas por medio de un proyecto pedagógico de aula de Meza y Rojas (2017), buscaba caracterizar y evaluar las prácticas de la comunicación no verbal. Este trabajo investigativo es de tipo descriptivo, los instrumentos de recolección de datos son la observación y el cuestionario. Según la investigación un porcentaje superior a la mitad de la población expone algunos hábitos con respecto al manejo de la CNV: utilizan objetos para disimular el movimiento de sus manos, tienen contacto visual frecuente con la audiencia y su volumen de voz no es adecuado, entre otros. Se enfatiza en la necesidad de desarrollar la $\mathrm{CNV}$ no solo en el área de lengua castellana sino integrarla en otras materias. Este hallazgo es fundamental en la articulación de lo observado por anteriores estudios, el engranaje entre la CNV y el saber disciplinar es imperativo. No obstante, esto debe encontrar justificaciones más certeras en el análisis y la interpretación que tienen los agentes del proceso educativo sobre la $\mathrm{CNV}$ para partir de una sensibilización que pueda garantizar la articulación entre diversas disciplinas y la comunicación corporal.

\section{La comunicación no verbal en el aprendizaje de lengua extranjera}

En el artículo La comunicación no verbal en la enseñanza del inglés como segunda lengua de Álvarez (2002) tenía como objetivo analizar la relevancia que tienen los elementos de la CNV en este proceso de aprendizaje. Así entonces, se efectuó una encuesta a 60 personas que contemplaban aspectos kinésicos y de paralenguaje; además, este instrumento era efectuado en dos partes distintas, una en inglés y otra en español. Dentro de los objetivos estaban: contrastar el uso de la CNV en ambas lenguas y explorar una relación posible entre fluidez verbal y no verbalidad. Como resultado se encuentra que se hace un mayor uso de la CNV en la L2 en el intento de transmitir un mensaje; no obstante, en este proceso se generan los falsos amigos kinésicos. Estos se comprenden como recursos no verbales de la lengua materna en la lengua extranjera se creen erróneamente universales. La contribución de este trabajo es vital para comprender la importancia de la CNV en el aprendizaje de lengua extranjera, sin duda, deja entrever un reto para cada docente en cuanto el abordaje de esta en el aula. El estudio podría haber explorado las concepciones del profesorado sobre la CNV, ya que, en caso de no considerarse relevante no puede incluirse en la didáctica de la lengua y por tanto, los errores kinestésicos permanecerán en las prácticas de estudiantes.

Asimismo, en el texto El lenguaje corporal en el proceso de enseñanza-aprendizaje de la lengua extranjera: inglés de Paternain (2016), se efectúa un análisis sobre el manejo del lenguaje corporal en dos casos. El primer caso tuvo lugar con 25 niños de grado 4 del colegio Calasanz de Pamplona; a partir de las dinámicas en clase se logra determinar que la CNV de cada docente influye positivamente en los procesos de comprensión y memoria del cuerpo estudiantil. Entretanto, el segundo caso, llevado a cabo con niños de grado 3, pretendía demostrar que el uso de la CNV por parte del alumnado como repaso para un examen se traduciría en notas más altas. Sin embargo, tras la aplicación, se demuestra la reducción de la nota tras el repaso no verbal. En conclusión, se manifiesta la importancia del uso de la CNV desde el personal docente y que la falta de trabajo acerca del uso del lenguaje no verbal con el estudiantado hace de su puesta en práctica un ejercicio contraproducente. Si bien este trabajo examina las implicaciones pedagógicas del uso de la CNV en el aula de lengua extranjera, no se explicita una relación directa frente a esta y el saber disciplinar, ni las concepciones que permean esta relación. 
En el artículo Nonverbal Teacher-student Communication in the Foreign Language Classroom de Pan (2014), se pretende explorar la comunicación entre los educandos y el conjunto docente en el marco del aprendizaje de lenguas. Para ello, se llevó a cabo un proceso de observación en una clase de inglés de primer año en una colegiatura. Se interpretaron aspectos de orden proxémico, tales como la postura y la dirección de la mirada. Así entonces se expone una organización espacial del salón tradicional que limita la comunicación entre docente-estudiante. También, la postura puede caracterizarse a partir de estos elementos: inseguridad, diferencias culturales y el intento por no caer en contradicción. Además, el contacto visual cada estudiante hacia su respectivo docente es muy limitado. Se concluye que un uso dinámico de la CNV en el aula hace del aprendizaje de lengua un proceso vívido. La aportación de este trabajo reside en indicar cómo la interacción, no solo puede optimizar la comunicación entre docente-estudiante, también crear conocimiento cultural sobre las técnicas específicas que esta involucra en una cultura distinta, de algún modo completa lo postulado por el trabajo anterior. No obstante, la necesidad de educar en la CNV de una cultura diferente se enuncia sin explorar el conocimiento previo o las conceptualizaciones de docentes y estudiantes sobre esta.

En conclusión, los trabajos revisados en las tres categorías: el cuerpo en la escuela, la CNV en el aprendizaje y la CNV en la lengua extranjera insisten en la necesidad de: reconceptualizar el cuerpo en cuerpo en la escuela más allá del orden disciplinar y funcional, educar en la CNV integrándola con los saberes disciplinares y la relevancia de la CNV en la lengua extranjera como catalizador de aprendizaje cultural. Sin embargo, considerando la revisión efectuada, ninguno de estos estudios articula las tres categorías, desde la conceptualización del cuerpo en la escuela hasta su impacto en contexto específico del aprendizaje de lenguas. De ahí que el presente estudio pretenda dar cuenta de las dinámicas entre la CNV y el aprendizaje de EFL (English as a Foreign Language) a través de la observación de experiencias en actividades de clase. Asimismo, se exponen las perspectivas que tienen las estudiantes sobre: la educación sobre el cuerpo, el cuerpo y la CNV en el aprendizaje y su impacto en el aula de lengua extranjera. Se considera que cualquier reconceptualización sobre el cuerpo, la CNV y el aprendizaje debe partir de examinar las configuraciones que hacen los educandos sobre estos elementos y cómo estas han sido posiblemente influenciadas por la escuela.

\section{Tipo De Investigación}

La presente investigación se alinea al paradigma cualitativo, ya que se busca descubrir y hacer parte de la construcción de un cuerpo de conocimiento a partir de las interacciones y complejidades de un contexto social, en este caso, educativo (Bisguerra, 2009). Así pues, se selecciona el método del estudio de caso con el propósito de analizar e interpretar las concepciones y prácticas sobre el cuerpo en la escuela, así como, sus posibles relaciones con la comunicación no verbal en el marco de la clase de lengua extranjera.

\section{Metodología}

A partir de este estudio de caso se generaron datos relacionados con las concepciones y prácticas sobre el cuerpo y sus posibles relaciones con el aprendizaje de inglés como lengua extranjera. Luego de un período de observación de las relaciones entre la comunicación no verbal, el cuerpo y las estudiantes, se diseñan tres preguntas de reflexión a partir de las cuales se obtuvo información referente al fenómeno.

Preguntas de reflexión.

¿Cómo educa la institución educativa sobre la comunicación no verbal y el cuerpo?

¿Qué relaciones proponen las estudiantes sobre la comunicación no verbal en el entorno escolar y en el aula de lengua extranjera?

¿Qué relevancia tiene la comunicación no verbal en el aprendizaje de lengua extranjera?

Unidades de análisis 
- Las prácticas y concepciones sobre el cuerpo en la escuela

El cuerpo

- La comprensión del cuerpo como comunicador.

- Posibles relaciones entre las concepciones y prácticas sobre el cuerpo en la escuela y el aprendizaje de lengua extranjera

\section{Métodos e instrumentos de recopilación de información}

Con el propósito de recolectar y sistematizar los datos obtenidos a través de las preguntas de reflexión se utilizaron instrumentos de recopilación de información tales como: la observación, entrevistas semiestructuradas y cuestionarios.

\section{Método de análisis de la información}

A partir de los datos recogidos en el ítem anterior se establece un grupo de categorías a partir de patrones otorgados a partir de las preguntas de reflexión. De modo que, esto en articulación a la evidencia hace posible la estructuración de conclusiones.

\section{Sujetos participantes}

El estudio de caso se enfocó en el contexto de un colegio femenino de carácter católico, localizado en Bogotá, Colombia. Los sujetos escogidos fueron 42 estudiantes de grado décimo entre los 14 y los 17 años. La elección de los participantes se justifica a partir de identificación de dificultades enfrentadas por las estudiantes en el uso del cuerpo y la comunicación no verbal durante la clase de lengua extranjera. Se observó que las jóvenes expresaban temor e incomodidad al deber presentar actividades que conllevasen el uso del cuerpo.

\section{Proceso de muestreo}

Los datos recopilados con base al cuestionario fueron tabulados, de manera que fue posible obtener porcentajes de acuerdo con las respuestas. Por su parte, las entrevistas semiestructuradas fueron grabadas y luego transcritas. En ambos instrumentos se seleccionaron palabras y frases que fueron constantes en las opiniones dadas por las estudiantes, con el propósito de configurar las variables en relación con cuerpo, educación, aprendizaje, comunicación no verbal y aprendizaje de EFL.

\section{ANÁLISIS Y DISCUSIÓN DE RESULTADOS}

\section{Descripción de experiencia (Observación)}

Este documento pretende dar cuenta de algunas experiencias con base a un proceso de aprendizaje de lengua inglesa y su relación la comunicación no verbal en el contexto escolar. La población consiste en un grupo de estudiantes de grado décimo pertenecientes a un colegio femenino de la ciudad. Durante el año se han observado algunos elementos problemáticos entre el ejercicio de las habilidades de producción y el despliegue 
corporal de las estudiantes. A su vez, se pretende explorar el rol de la institución en la educación desde el cuerpo y su relación con este fenómeno en el aula.

El primer escenario consistió en la creación de un Flea Market por parte de las estudiantes. Se otorgaron los parámetros de su elaboración a partir de criterios básicos: vocabulario para la compra y venta de artículos, conocimiento histórico del artículo y el montaje dentro un espacio al aire libre con los objetos. En consecuencia, las estudiantes demostraron un gran interés en la actividad lo cual se reflejó en su práctica lingüística: lograron construir discurso desde los objetos y utilizar el vocabulario de modo funcional.

Entretanto, en la interacción con el espacio y la docente se evidenció que las estudiantes manejaban una prosémica distante, demostraban una postura recogida y la escogencia de un plano inferior frente al interlocutor. Por otro lado, en algunos casos se pretende memorizar la historia del producto, de modo que, al olvidar algún concepto se perdía el hilo discursivo. De esto puede inferirse que el objeto se distancia de la información lingüística que lo describe, el producto y su información referencial que no se vinculan para generar una situación comunicativa en la compra y venta.

Con el fin de explorar un segundo espacio de interacción se eligió el restaurante; las estudiantes debían pedir la cuenta, ordenar comida, ofrecer la carta, entre otros. A pesar de algunas dificultades de pronunciación, la práctica lingüística fue satisfactoria en la medida que pudieron describir la comida, dialogar sobre otras temáticas ajenas al contexto, utilizar excusas para rechazar un alimento, entre otros.

$\mathrm{Al}$ mismo tiempo, se observó que la actividad no se concibe como una presentación para la totalidad del grupo, aunque el salón haya sido organizado en mesa redonda. La presentación fue dirigida hacia la docente al punto que se dio la espalda al público. En consecuencia, la posibilidad de efectuar un proceso de aprendizaje articulado con los distintos grupos de trabajo a través de la escucha se desaprovecha.

En un tercer momento se planteó la creación de un alter ego dándoles a escoger entre un cómic o una presentación oral. El trabajo tenía dos objetivos: la apropiación gramatical de gerundios e infinitivos y la construcción de un personaje. En ambos grupos, la mayoría de las jóvenes decidió escribir el cómic. De esto puede interpretarse que existe cierto temor a comunicar con el cuerpo porque, tal vez, se carece de un proceso educativo que reconozca al cuerpo como creador de significado.

De hecho, la actividad oral reveló que, si bien había una inventiva de personajes, la parte lingüística se desliga de la expresión corporal y de elementos semióticos como el vestuario. Se mostró una carente teatralización del personaje: el horizonte de sentido desde el cuerpo fue de cierre, el vestuario no fue empleado acorde a las subjetividades descritas y la audiencia tampoco fue tomada en consideración. De nuevo, se alcanzó el objetivo gramatical, en contraposición de un objetivo comunicativo no verbal.

Paradójicamente, a través del cómic las jóvenes demostraron tener notables fortalezas en la estructuración de códigos diversos en el texto. Hay una correlación entre el código visual y escrito que se demuestra en el manejo del color, la forma, la distribución de espacios y los enunciados, entre otros. Incluso, se retratan figuras retóricas como: el sarcasmo, el chiste, la ironía. Del mismo modo, los personajes del cómic demostraron complejidades subjetivas en su carácter y actuar, tal como se aspiraría en la creación literaria.

Ahora bien, durante esta experiencia pedagógica se entiende que las estudiantes estas inmersas en un marco institucional, cuyas normatividades respecto a la comunicación verbal y no verbal se ajustan a un prototipo de la feminidad. En el colegio, son constantes las órdenes y los pedidos para que las jóvenes utilicen un vocabulario adecuado y limiten su espacio corporal en espacios diversos. Se arguye que ellas, como mujeres, no deben tener ciertas posturas, utilizar cierto maquillaje, expresarse de cierta manera. Por supuesto, esto genera numerosos cuestionamientos por parte de las jóvenes en relación con sus posibilidades de expresión y las reglas impartidas desde la coordinación.

Así pues, hay un desfase frente a lo que se requiere desde el aula de lenguas extranjeras y las disposiciones institucionales. En el aprendizaje de lenguas se pretende desarrollar un acercamiento vivido e incluyente, es decir, entender que este proceso no solo acoge la estructuración formal de la lengua sino sus componentes extralingüísticos. Con esto, crear una experiencia de aprendizaje significativo que involucre distintos modos 
de significación: el cuerpo, la imagen, lo auditivo. No obstante, desde el colegio se establecen parámetros reducidos frente a las posibilidades de comunicativas desde dichos modos.

\section{Concepciones de las estudiantes sobre el cuerpo y la CNV mediadas por las normas y el aprendizaje}

Con el propósito de conocer las relaciones que establecen las estudiantes entre el cuerpo, la CNV con las normas del colegio y el aprendizaje se llevó a cabo un cuestionario a 42 estudiantes de grado décimo. Se elige esta población con base a los fenómenos descritos en sobre la articulación entre el desarrollo de las habilidades de producción y la comunicación no verbal. Asimismo, a partir de la observación de las dinámicas en la institución se identifica una permanente inconformidad de las estudiantes con respecto a las normativas que limitan la expresión y comunicación con el cuerpo. De modo que, en el cuestionario se efectúan las siguientes preguntas:

\section{Cuestionario:}

1. ¿Qué opina de las normativas institucionales respecto a la comunicación no verbal?

2. ¿Cree que la CNV influye en la parte académica? ¿Por qué?

3. ¿Cree que la CNV es relevante en el aprendizaje de lengua extranjera? ¿Cómo?

\section{Análisis de la pregunta 1}

Se configuran cinco categorías en las cuales se sintetizan las opiniones de las estudiantes en relación con la funcionalidad de la normativa y su incidencia sobre la CNV tal como pueden verse en la siguiente Tabla:

TABLA 1

1. Relaciones posibles entre la normatividad institucional y la comunicación no verbal

\begin{tabular}{|l|l|}
\hline Categoría & Código \\
\hline $\begin{array}{l}\text { Intrascendencia de la normatividad sobre el cuerpo en } \\
\text { el proceso académico Importar lista }\end{array}$ & INT NORC AC \\
\hline $\begin{array}{l}\text { La normatividad sobre el cuerpo no permite la } \\
\text { expresión subjetiva Importar lista }\end{array}$ & NORC NPE \\
\hline $\begin{array}{l}\text { La normatividad sobre el cuerpo es formativa Importar } \\
\text { lista }\end{array}$ & NORC FORM \\
\hline $\begin{array}{l}\text { La Intrascendencia de la norma y la limitación de la } \\
\text { expresión obedecen a un prototipo de feminidad. } \\
\text { Importar lista }\end{array}$ & NORC PROT FEM \\
\hline
\end{tabular}

Fuente: Elaboración propia.

De las cuatro categorías mencionadas en la Tabla 1 se obtiene que la mayoría de las estudiantes considera que las normatividades sobre el cuerpo dentro de la institución no son relevantes frente a su proceso académico. Es decir, las jóvenes no identifican o comprenden los argumentos que fundamentan las normativas sobre el cuerpo. Asimismo, las estudiantes no reconocen la relación entre estas disposiciones y su proceso académico, rendimiento en las materias, construcción de conocimiento en el aula, aprobación de procesos evaluativos. 
De igual modo, cerca de un cuarto de las estudiantes asocian la normativa sobre el cuerpo a una restricción sobre su expresión subjetiva (ver Tabla 1). En otras palabras, las reglas sobre la comunicación no verbal no les permiten dar a conocer sus sentimientos y actitudes de forma espontánea, ya que, según ellas, se busca homogenizar el comportamiento de las jóvenes. En este respecto, se encuentran dos minorías de estudiantes que racionalizan el proceder institucional de dos modos: el colegio restringe el uso de la CNV debido a que desarrolla un proceso de formación sobre el cuerpo, dando origen a la siguiente categoría (ver Tabla 1). En segundo orden, la institución demanda el cumplimiento de la normativa porque pretende promover un estereotipo sobre la femineidad en ellas (ver Tabla 1). Por un lado, se parte del hecho que la institución regula la CNV de las estudiantes para prepararlas a enfrentar un contexto social, en el cual son requeridos ciertos patrones de interacción y comportamiento que limitan la expresión subjetiva. Por su parte, hay jóvenes que señalan que el colegio se identifica con una visión convencional sobre lo que significa ser mujer: delicadeza, recato, cierre corporal, pasividad; y se propone que las estudiantes hagan práctica este estereotipo sin considerar otros modos de comprender que es ser mujer y problematizarlos.

Cabe mencionar que algunas de las normativas sobre el cuerpo en la institución son:

Se prohíbe el uso de piercing o tatuajes puesto que pueden atentar contra la salud e influyen negativamente en el proceso formativo de la estudiante

No se permiten los tintes ni cortes extravagantes puesto que atentan contra la filosofía institucional, se busca la formación de una mujer delicada y femenina.

El uniforme de la institución debe portarse de manera adecuada, completo y limpio.

No se permite el uso de manillas, esmaltes de color oscuro, aretes grandes, adornos excesivos, joyas ostentosas puesto que restan presentación al uniforme.

La postura en los eventos institucionales debe ser recta sin recostarse demostrando respeto y atención.

No está permitido recostarse en el prado de la institución.

Como se ha indicado, las estudiantes no comprenden el argumento que respalda las normas en el espacio institucional. Según ellas, elementos como el maquillaje, los tintes, manillas, peinados son irrelevantes en su aprendizaje. De este modo, la aplicabilidad de la norma se da en la medida que su incumplimiento trae consigo una sanción convivencial. Hay un desface entre la formación que pretende dar la institución y la voluntad del estudiantado.

En cuanto a la segunda categoría, las jóvenes se manifiestan en contra de la norma porque no les permite expresarse. Señalan que ellas deben tener libertad sobre lo que quieren hacer con su cuerpo. Se comprende el cuerpo como una propiedad donde recae un derecho individual. Así que, el cuerpo, según las estudiantes es un dominio individual y no parte de una construcción comunitaria o cultural.

Un tercer grupo de la población indica que está parcialmente a favor de las normas puesto que estas suponen un comportamiento adecuado. Algunas de las jóvenes señalan cada una de esas reglas nos forma para un futuro .creo que (las perforaciones, joyas, tintes) no es adecuado para un colegio, porque lo primero que ven en ti es tu apariencia física, ya que esto da mucho que pensar de las personas" (sujeto 13, comunicación personal, 3 de febrero de 2017). Se identifica que hay un respaldo a ciertas creencias sociales deterministas que clasifican a partir de convenciones sociales lo valido y no válido en el uso de elementos semióticos sobre el cuerpo.

En la última categoría se articulan la primera y la segunda en razón a la búsqueda de un prototipo de feminidad. Las estudiantes critican este elemento preguntándose sobre la validez de una norma que refleja un estereotipo cultural sobre el ser mujer. "En esta institución tienen muy catalogados el cómo debería ser una mujer, una mujer debe sentarse bien porque es una mujer, no se deben utilizar piercing porque una mujer no se ve bien"(sujeto 21, comunicación personal, 6 de febrero de 2017). De modo que, las estudiantes demuestran una necesidad por construir una conceptualización de mujer distinta a la ofrecida en la institución, aunque, no se sabe cómo hacerlo y es un tema poco abordado en los espacios académicos. En otras palabras, se identifica que la comunicación a partir del cuerpo desde las estudiantes no puede seguir siendo sinónimo de un prototipo con el cual, según ellas, no se sienten identificadas. 


\section{Análisis pregunta 2}

Se configuran 4 categorías respecto a la importancia de la CNV en el proceso de aprendizaje como puede verse en la siguiente Tabla 2:

TABLA 2

2. Relaciones posibles entre el aprendizaje y la comunicación no verbal

\begin{tabular}{|l|l|}
\hline Categoría & Código \\
\hline $\begin{array}{l}\text { La CNV no es relevante en el aprendizaje } \\
\text { Importar lista }\end{array}$ & CNV NRA \\
\hline $\begin{array}{l}\text { La relevancia en el aprendizaje depende del } \\
\text { tipo de CNV Importar lista }\end{array}$ & TCNV R \\
\hline $\begin{array}{l}\text { La CNV es relevante en cuanto envia } \\
\text { mensajes Importar lista }\end{array}$ & CNV REM \\
\hline $\begin{array}{l}\text { La CNV es relevante en la comunicación } \\
\text { docente-estudiante. Importar lista }\end{array}$ & CNV CDE \\
\hline
\end{tabular}

Fuente: Elaboración propia.

De acuerdo con la Tabla 2 la primera categoría, la cual corresponde a más de la mitad del grupo, se manifiesta que la comunicación no verbal no es relevante en el proceso de aprendizaje. Esto se explica en la medida que la CNV se comprende por las estudiantes desde las normas institucionales sobre el cuerpo expuestas en el ítem anterior. Según las estudiantes la CNV: "no influye porque el hecho de que tenga el cabello largo, mono, rojo, una perforación o que uno se siente derecho o recostado no tiene mayor relación con la manera en la que uno aprende, o no..." (sujeto 17, comunicación personal, febrero 19 de 2017). En consecuencia, la conceptualización de la CNV se limita y no permite su exploración desde los gestos, la kinésica, proxémica, entre otros de sus elementos constitutivos.

En cuanto a la segunda categoría (ver Tabla 2), las jóvenes exponen que la relevancia de la CNV está condicionada por su tipo. Es decir, se argumenta que hay dos tipos básicos de CNV: la que involucra el movimiento (gestual-postura-proxémica) y la estética en el cuerpo. Por un lado, las estudiantes señalan que los gestos que se ejecutan durante una exposición o en la interacción con el cuerpo docente sí son relevantes, ya que, pueden reforzar lo que se dice o pueden demostrar interés o apatía en el aula. Como muestra:

Como lo mencioné anteriormente un piercing o un tinte no afecta en el aprendizaje, pero en cuanto a la postura corporal creo que sí, porque en muchas materias se necesita hablar en público y hay que tener maneras o posturas adecuadas frente al público, porque eso también ayuda a transmitir cosas en público. (Sujeto 15, comunicación personal, febrero 20 de 2017)

En este orden de ideas, en las estudiantes agrupadas en esta categoría hay una preocupación por presentarse en el espacio del aula, de acuerdo con parámetros comunicativos que les permita validar lo que enuncian verbalmente. No obstante, al igual que en el ítem anterior, hay una negación a considerar el maquillaje, los tintes, piercing y demás como parte de la comunicación que pueden interpretarse en el aula y que intervienen en la interacción con el otro.

Por otra parte, un grupo minoritario de la población concluye que la CNV sí es relevante en la medida que envía mensajes al receptor en medio de la situación comunicativa (ver Tabla 2). Según ellas, la funcionalidad de la CNV está en el deseo de darse a entender y expresarse frente a quien escucha. Por ejemplo: "Estoy de acuerdo con la CNV porque es una manera de dar a entender...y también es la oportunidad que el receptor lo interprete de acuerdo con sus ideas" (Sujeto 33, comunicación personal, marzo 5 de 2017). De igual modo, las jóvenes enuncian que a partir de la CNV ellas pueden manifestar actitudes frente a distintos espacios y situaciones. 
En cuanto a la última categoría pocas alumnas construyen una relación entre la CNV y la interacción entre docente y estudiante (ver Tabla 2). Se articula con la anterior en la medida que las jóvenes reconocen que se demuestran disposiciones y actitudes en el aula gracias a elementos no verbales. Sin embargo, también reconocen que ellas pueden dar cuenta de que no comprendieron la temática de clase a partir de esta, por supuesto, esto conduce a que el personal docente, identificada la duda, vuelva a explicar. De igual manera, algunas jóvenes indican que sus docentes hacen uso de estos recursos para que ellas entiendan mejor.

\section{Análisis de la pregunta 3}

En la indagación sobre la importancia de la CNV en la lengua extranjera según las jóvenes se configuran las siguientes categorías en la Tabla 3:

TABLA 3

3. Relaciones posibles entre la comunicación no verbal y el aprendizaje de la lengua extranjera

\begin{tabular}{|l|l|}
\hline Categoría & Código \\
\hline $\begin{array}{l}\text { 3.1. La CNV no es relevante en el aprendizaje } \\
\text { de lengua extranjera }\end{array}$ & CNV NR LE \\
\hline $\begin{array}{l}\text { 3.2. La CNV es relevante en Cuanto envía } \\
\text { mensajes }\end{array}$ & CNV REM \\
\hline $\begin{array}{l}\text { 3.3. La CNV es relevante en la comunicación } \\
\text { docente-estudiante }\end{array}$ & CNV CDE \\
\hline 3.4. No hay seguridad sobre el tema & NST \\
\hline $\begin{array}{l}\text { 3.5. La CNV es distinta en la cultura } \\
\text { extranjera }\end{array}$ & CNV CE \\
\hline 3.6. La CNV es parte de estilos de aprendizaje & CNV EA \\
\hline
\end{tabular}

Fuente: Elaboración propia.

Como puede evidenciarse en la Tabla 3, algunas de las categorías de la pregunta anterior se repiten en esta. Son constantes las categorías CNV NR, CNV REM, CNV CDE.

No obstante, en relación con la comunicación no verbal no relevante en el proceso de aprendizaje se tiene una reducción considerable. Este fenómeno se explica en la medida que, en el marco de la clase se ha implicado la importancia de la CNV en exposiciones, diálogos, dramatizaciones. De manera que, construyen significado junto con lo expresado verbalmente. No obstante, este aspecto no es lo suficientemente contundente para desmitificar la irrelevancia de la CNV en el aprendizaje de lenguas.

Una de las razones constantes por las cuales se afirma la irrelevancia de la CNV en el aprendizaje de la lengua extranjera obedece a una perspectiva cognitiva del proceso. Como muestra de ello, según una joven la CNV: "no me parece relevante ya que el tener elementos estéticos o no, no afecta tu capacidad de pensar"(Sujeto 30, comunicación personal, marzo 29 de 2017). Asimismo, se ratifica el resultado de la pregunta dos, en cuanto a la conceptualización de la CNV está guiada por elementos estéticos en relación con las normativas institucionales.

Ahora bien, sobre la tercera categoría CNV en la comunicación docente-estudiante (ver Tabla 3) pasa de ser poco relevante en la pregunta a tener un impacto significativo en la pregunta 3. Este fenómeno puede deberse a que el estudiantado de segunda lengua tiene una mayor necesidad de darse a entender frente al grupo docente. Sin embargo, el educando asume ciertas formas no verbales configuradas en su cultura y que no siempre corresponden a la cultura extranjera. Por otro lado, puede influir la interacción que se ha dado en el aula con la docente de lengua inglesa, quien en algunas ocasiones, pone de relevancia la CNV como esencial en la expresión y es ella quien elabora el cuestionario. 
Entretanto, aparece una categoría nueva en la cual la joven expresa no estar segura si la CNV es relevante en el aprendizaje de lengua extranjera (ver Tabla 3). Esto se debe a que hay un encuentro entre los elementos estéticos discutidos desde la norma y el lugar que tienen otras manifestaciones de lo no verbal en el aula. De modo que, esto genera confusión en las estudiantes quienes no saben cuál de las dos conceptualizaciones deben considerar. Esto se relaciona con los hallazgos de la pregunta 2 y su segunda categoría sobre los tipos de CNV.

A pesar de ser minoritarias, las dos últimas categorías presentan elementos nuevos e interesantes: La CNV en el marco de la cultura extranjera y los estilos de aprendizaje. Sobre el primero se indica que: "Aparte los distintos idiomas tienen distintos acentos y estos vienen con expresiones faciales y corporales diversas, algunas muy marcadas y otras poco, lo importante es la simbología que tienen estos gestos para estas personas a diferencia de nosotros"(Sujeto 30, comunicación personal, marzo 14 de 2017).

Esto es vital en la medida que, hay un segmento reducido de estudiantes que comprende la diferenciación cultural de la CNV y su importancia en el proceso de aprendizaje. Se ve entonces a la lengua como una posibilidad de conocimiento que cultural que articula expresiones verbales y no verbales. Por su parte, hay se menciona a la CNV como una posibilidad de aprender de manera diferente, ya que, según la estudiante no todos aprenden igual, habrá quienes aprendan a partir de gestos o movimientos.

\section{Conceptualizaciones sobre el cuerpo, el aprendizaje, la CNV y la educación corporal}

Con el propósito de indagar de forma más detallada sobre las conceptualizaciones y relaciones que efectúan las estudiantes sobre estos conceptos se llevaron a cabo cinco entrevistas semiestructuradas Estas entrevistas contaron con la participación de cinco de las jóvenes que efectuaron el cuestionario. Dos de las jóvenes fueron escogidas debido a su posición crítica frente a las disposiciones del manual de convivencia, otras dos estudiantes con una opinión cercana a la neutralidad sobre el tema: dan a conocer pros y contras sobre el este, y una estudiante con una postura institucional. Esto tenía como propósito descubrir posibles discrepancias o puntos de encuentro en relación con las preguntas y así identificar qué elementos son constantes en las perspectivas de las estudiantes para ser entendidos como conceptualizaciones sobre el cuerpo, el aprendizaje, la CNV y la educación corporal.

Las preguntas fueron:

1. ¿Qué es el cuerpo?

2. ¿Qué significa aprender?

3. ¿Cómo se educa el cuerpo en la institución educativa?

4. ¿Qué es la CNV?

5. ¿Crees que es posible comunicar con el cuerpo? ¿Cómo?

De acuerdo con las respuestas dadas por las estudiantes se configuran las siguientes categorías según cada pregunta:

1. Conjunto de partes que forman a un ser vivo.

2. Adquisición de conocimiento

3. Falta de educación sobre el cuerpo en la institución

4. Comunicación sin uso de palabras de la expresión y la identidad

5. Comunicación con el cuerpo: Reacción física, sentimental e inconsciente. 


\begin{tabular}{|c|c|c|c|c|c|}
\hline \multicolumn{6}{|c|}{ Ejes temáticos de las preguntas } \\
\hline $\begin{array}{l}\text { Sujetos } \\
\text { entrevistados }\end{array}$ & Cuerpo & Aprender & $\begin{array}{l}\text { Educación } \\
\text { sobre el cuerpo }\end{array}$ & $\begin{array}{l}\text { Comunicación } \\
\text { no verbal }\end{array}$ & $\begin{array}{l}\text { Posibilidad } \\
\text { del cuerpo } \\
\text { como } \\
\text { comunicador }\end{array}$ \\
\hline Sujeto 2. & $\begin{array}{l}\text {-Lo que tienen } \\
\text { las personas y } \\
\text { les permite } \\
\text { tener vida }\end{array}$ & $\begin{array}{l}\text {-Adquirir } \\
\text { conocimiento } \\
\text { s }\end{array}$ & $\begin{array}{l}\text {-No enseñan lo } \\
\text { que implica sino } \\
\text { las partes }\end{array}$ & $\begin{array}{l}\text {-Darse a } \\
\text { entender sin } \\
\text { palabras }\end{array}$ & $\begin{array}{l}\text {-Reacciones } \\
\text { físicas: las } \\
\text { personas } \\
\text { tiemblan o } \\
\text { se les pone } \\
\text { la piel de } \\
\text { gallina }\end{array}$ \\
\hline Sujeto 33. & $\begin{array}{l}\text {-Conjunto del } \\
\text { todo }\end{array}$ & $\begin{array}{l}\text {-Adquirir } \\
\text { conocimiento } \\
\text { s y aplicarlos } \\
\text { en la vida }\end{array}$ & $\begin{array}{l}\text {-Educan a partir } \\
\text { de una serie de } \\
\text { reglas. }\end{array}$ & $\begin{array}{l}\text {-Transmisión } \\
\text { de lo que } \\
\text { pensamos o } \\
\text { sentimos }\end{array}$ & $\begin{array}{l}\text { Transmisión } \\
\text { de } \\
\text { sentimientos }\end{array}$ \\
\hline Sujeto 25. & $\begin{array}{l}\text {-Las partes que } \\
\text { conforman al } \\
\text { ser humano }\end{array}$ & $\begin{array}{l}\text {-Adquirir } \\
\text { conocimiento } \\
\text { s }\end{array}$ & $\begin{array}{l}\text {-No se educa } \\
\text { sino a partir de } \\
\text { la norma }\end{array}$ & $\begin{array}{l}\text {-Lo que no } \\
\text { puedo } \\
\text { transmitir con } \\
\text { palabras }\end{array}$ & $\begin{array}{l}\text { - gestos, } \\
\text { decir algo } \\
\text { más. }\end{array}$ \\
\hline Sujeto 27. & $\begin{array}{l}\text { Las partes que } \\
\text { conforman al } \\
\text { ser. }\end{array}$ & $\begin{array}{l}\text {-Adquirir } \\
\text { conocimiento } \\
\text { s }\end{array}$ & $\begin{array}{l}\text { - No se educa } \\
\text { sino a partir de } \\
\text { la norma } \\
\text { basada en un } \\
\text { prototipo de } \\
\text { feminidad }\end{array}$ & $\begin{array}{l}\text {-Gestos y } \\
\text { vestir: lo que } \\
\text { enuncia el ser } \\
\text { de sí mismo }\end{array}$ & $\begin{array}{l}\text {-Expresión } \\
\text { inconsciente }\end{array}$ \\
\hline Sujeto 31. & $\begin{array}{l}\text {-Las partes que } \\
\text { conforman al } \\
\text { ser. }\end{array}$ & $\begin{array}{l}\text {-Adquirir } \\
\text { conocimiento } \\
\text { s y aplicarlos } \\
\text { en la vida }\end{array}$ & $\begin{array}{l}\text { - No se educa } \\
\text { sino a partir de } \\
\text { la norma } \\
\text { basada en un } \\
\text { prototipo de } \\
\text { feminidad }\end{array}$ & $\begin{array}{l}\text {-Todos los } \\
\text { gestos que } \\
\text { comunican lo } \\
\text { que somos }\end{array}$ & $\begin{array}{l}\text {-Expresión } \\
\text { de } \\
\text { sentimientos } \\
\text { sin decir } \\
\text { palabra. }\end{array}$ \\
\hline
\end{tabular}

TABLA 4

4. Concepciones de las estudiantes frente al cuerpo, el aprendizaje, las relaciones entre cuerpo e institución, la comunicación no verbal y el cuerpo como comunicador Fuente: Elaboración propia.

Como se describe en la Tabla 4, la conceptualización del cuerpo se lleva a cabo desde un modelo biológico y taxonómico. Para cuatro de las entrevistadas cuerpo es una composición de partes del ser, lo que permite la vida; una de ellas lo define como algo que se posee. Estas concepciones sobre el cuerpo concuerdan con lo señalado por el estudio El cuerpo en Colombia: Estado del arte cuerpo y subjetividad en el cual se indica la carencia de una construcción de significado a partir del cuerpo que lo entienda más allá de su actividad motriz. Claro está, que el cuerpo es materialidad orgánica; sin embargo, la exploración del cuerpo experiencial, epistémico, simbólico y comunicador no se ha tenido cabida en el aula.

Entretanto, todas las estudiantes afirman que el aprendizaje es adquirir conocimientos, además, dos de ellas enfatizan en la aplicabilidad de este. No obstante, en ningún caso se hizo una articulación entre el aprender y el concepto anterior, el cuerpo.

Por su parte, en cuanto a la educación del cuerpo dentro de la institución, 4 de las entrevistadas arguyen que las normatividades son el instrumento que media entre el colegio y el cuerpo. En 3 de los casos se afirma que el cuerpo no se educa sino se regula. Por el contrario, una de las estudiantes indica que se educa a partir de la norma. De modo que, hay una ambigüedad en cuanto a la conexión entre la educación y la norma, en la cual no se tiene claridad si educar incluye o no dicha reglamentación.

Así entonces, en cuanto a la educación corporal este estudio valida los trabajos investigativos revisados que indican: la disciplinariedad sobre el cuerpo en la escuela y la necesidad de una educación interdisciplinar sobre este. Las prácticas sobre el cuerpo se guían sobre una base comportamental la cual establece actos válidos o inválidos desde el cuerpo, premia o sanciona según ellos mismos.

En contraposición a lo señalado en el texto El cuerpo en la Escuela de Scharagrodsky (2007), los parámetros binarios entre las acciones de hombres y mujeres mediadas por el cuerpo no han sido superados. Tanto en la observación como lo señalado por dos de las estudiantes en la entrevista, la aceptación de ciertos comportamientos se fundamenta en creencias estereotipadas sobre el género. Incluso, dentro de las normas de la institución está explicitado el deseo de formar una mujer delicada y femenina. Por supuesto, no se implica 
que las prácticas institucionales sobre este respecto son equivalentes a las dinámicas de la escuela hace 20 o 50 años. Las dinámicas se han transformado pero la fundamentación binaria entre los imaginarios de femenino y lo masculino continúa.

Por otro lado, las definiciones sobre la CNV son diversas, aunque con algunos puntos en común. Las jóvenes señalan que la CNV es hacerse entender los gestos expresar lo que sentimos. Esto indica que hay ambigüedad sobre la CNV y su funcionalidad en el aprendizaje y en la cultura. No se han considerado las aportaciones de la CNV en el aula como lo indican las investigaciones consultadas: la interacción docenteestudiante, el proceso de aprendizaje, el conocimiento cultural.

No obstante, las jóvenes incluyen en su conceptualización de la CNV elementos clave como los gestos, el vestir, lo que pensamos y sentimos, lo que somos. Estos aspectos son interesantes en la medida que las jóvenes ven en la CNV una posibilidad simbólica en articulación con su subjetividad. También, esto se soporta en sus definiciones y prácticas sobre el cuerpo y la institución. Para ellas, el cuerpo es una posibilidad expresiva sobre la cual la norma interviene.

Así pues, el cuerpo se reconoce como comunicador. Para la mayoría de las estudiantes de algo más o de sentimientos. En el caso de una de ellas, el cuerpo biológico también comunica. En coherencia con lo anterior, se denota un reconocimiento del cuerpo simbólico emocional y orgánico, sin embargo, embestido de ambigüedad.

\section{Conclusiones}

A la luz de los resultados, se identifican una serie de fenómenos relacionados con el estado de la cuestión, este estudio se alinea con lo indicado por otros trabajos en: la concepción biológica y disciplinar del cuerpo en la escuela, la poca relevancia de la comunicación no verbal en el aprendizaje disciplinar y la falta de exploración del cuerpo como comunicador en el aula de EFL. Esto se explica en el hecho que las prácticas en las instituciones siguen siendo regulatorias y coercitivas. Esto influye sobre las opiniones que forma el estudiantado sobre el cuerpo y la CNV, ya que, al reducírseles como dispositivos de control, no hay interés en su desarrollo ni en cómo puedan aportar la construcción de saberes disciplinares.

Hay una diversidad de normativas sobre el cuerpo que le indican cómo debe actuar y cómo debe verse. Sin embargo, estas regulaciones no encuentran mayor argumentación que en imaginarios sociales a partir de conceptos como delicadeza, feminidad, masculinidad, decencia, entre otros. En consecuencia, tal como lo señala Chavarriaga y Ochoa (2015), una gran cantidad de estudiantes ven en la norma la consolidación de estereotipos sobre el cuerpo que conflictúan con su posición subjetiva, por consiguiente, hay un desfase entre las creencias y actitudes de la escuela y las de los jóvenes. Paradójicamente, si bien y las estudiantes resisten frente a los estereotipos impuestos por la normativa, su conceptualización sobre el cuerpo es algo que se posee y ocupa un espacio y permite la vida. Así entonces, no hay una estructuración y articulación de argumentos sobre la definición del cuerpo y las normas por parte de las jóvenes.

De igual modo, se distinguen dos factores: la falta de valoración de la norma en las estudiantes y la incidencia de la norma en la conceptualización de la CNV. El primero, refiere que las jóvenes identifican que las reglas son irrelevantes en su proceso de aprendizaje o que les imposibilita expresarse. El segundo, señala que la CNV no tiene importancia en el proceso, ya que, la CNV equivale a pensar en los comportamientos no permitidos sobre el cuerpo. Es decir que, al igual que sucede con las conceptualizaciones sobre el cuerpo la CNV no es vista en relación con el aprendizaje y es dada por sentado sin establecer reflexiones sobre sus implicaciones expresivas, subjetivas y simbólicas, necesidad que Bárcena y Melich (2000) manifiestan en su estudio.

En efecto, la conceptualización y el alcance de la CNV en el aprendizaje consiste en un devenir exclusivo entre la normativa institucional y la resistencia a la normativa. La CNV es para ellas sinónimo de: pintarse el pelo, maquillarse, ponerse un piercing y por supuesto, estos elementos hacen parte de la no verbalidad. Con 
todo, estos y otros aspectos son posibilidades de comunicación que no podrían ser descalificados del proceso de aprendizaje y de la construcción de un carácter subjetivo cambiante.

Así pues, se presentaría la posibilidad para las jóvenes de leer e interpretar lo que ellas comprenden como expresiones de su subjetividad, se encuentra sujeta a la resistencia a las reglas institucionales y no a la posibilidad de expresar y aprender con el cuerpo. De modo que, esto les permita decidir de modo más reflexivo sobre cómo se presentan ante la otredad a partir de los discursos que les permean. En otros términos, la escuela partir de la CNV como una posibilidad de lectura de subjetividades en relación con el otro desde una comprensión de los discursos que mediatizan lo simbólico en el cuerpo.

Entretanto, la CNV se caracteriza por parte de las jóvenes como una posibilidad expresiva y de interacción. No obstante, la carencia en la didáctica del estudio de estos constitutivos genera ambigüedad sobre sus múltiples posibilidades de significación y comunicación. La CNV se asocia a los gestos, posturas, signos estéticos, lo que no se dice, algo más allá de las palabras, pero no hay una explicación sobre sus significación y funcionalidad en la comunicación. Ni la relación de la construcción de estos significados en la construcción de saber y la interacción con la complejidad que significa el encuentro con la otredad, sea entendida como otro sujeto, cultura o forma de ver el mundo.

De hecho, cuando se plantea a las estudiantes la lengua extranjera en conexión con la CNV su perspectiva tiene un ligero cambio. La CNV es relevante en cuanto envía mensajes de modo que, situar los elementos no verbales desde el aprendizaje de lengua genera una consciencia de la interacción en las jóvenes. Así pues, en la situación comunicativa no solo confluye lo lingüístico, ya que, la transmisión de elementos extralingüísticos es activada en cada relación con otros sujetos y las situaciones comunicativas derivadas de esta (Potayos, 2003). Por tanto, es de extrañar que los constitutivos de la CNV sean poco abordados en el aula de lengua materna y menos de segunda lengua. Con ello se desconoce un amplio espectro del encuentro con el otro.

El encuentro con la otredad involucra las particularidades de la cultura a la cual ella pertenece. Entonces, el aprendizaje de segunda lengua debe contemplar, tal como lo indica Álvarez (2002), aspectos no universales de la CNV. Es decir, en la interacción con la cultura extranjera deben conocerse sus elementos no verbales propios de la mano con sus especificidades lingüísticas. Esto involucra una didáctica de la lengua extranjera culturalmente sensible, en la cual el cuerpo se resignifique y sea vital.

De igual modo, la CNV en el marco del aprendizaje de lenguas no solo es una necesidad cultural sino la posibilidad de vivificar la lengua extranjera. Como se expuso durante las observaciones de este estudio, las estudiantes tienden a privilegiar la corrección lingüística sobre la comunicación con el cuerpo. Por supuesto, esto genera un saber elemental de la lengua extranjera, en el cual se descifra y memoriza el código según la tarea, pero no hay aprendizaje significativo. Dicho aprendizaje involucraría la agencia de cada estudiante sobre la lengua y la comprensión de la segunda lengua como un capital simbólico (Bourdieu, 1989) que le permita a las personas jóvenes expresarse y participar de forma verbal y extralingüística en las dinámicas de un mundo globalizado.

Finalmente, a partir de este estudio se desea proponer una resignificación del cuerpo y la CNV en el aprendizaje. El cuerpo como experiencia, expresión simbólica, comunicación. Esto permitiría interactuar con el otro de un modo más reflexivo, considerándolo en sus particularidades políticas, culturales, simbólicas, expresivas. Por supuesto, el aprendizaje de una lengua extranjera puede constituirse en una oportunidad para ello, encontrarse con la otredad no solo desde la palabra sino desde el gesto, la mirada, el espacio, el cuerpo.

\section{REFERENCIAS BIBLIOGRÁFICAS}

Álvarez, G. (2002). La comunicación no verbal en la enseñanza del inglés como segunda lengua. Philologia Hispalensis, 16(1), 7-18. https://dialnet.unirioja.es/servlet/articulo?codigo $=748273$

Arévalo, J. (2018). En torno al concepto de cuerpo desde algunos pensadores occidentales. Hallazgos, 9,119-131.

Bárcena, F. y Melich, J. (2000). El aprendizaje simbólico del cuerpo. Revista Complutense de Educación, 2(2), 59-81. 
Bisguerra, R. (2009). Metodología de la investigación educativa, Madrid, España: Editorial la Muralla.

Bourdieu, P. (1989). Social Space and Symbolic Power. Sociological Theory, 7(1). 14-25.

Bunglowala, A. (2015). Nonverbal communication: An integral part of teaching learning process. International Journal of Research in Advent Technology. (especial), 371-375.

Cabra, N y Escobar, M. (2014). El cuerpo en Colombia: Estado del arte cuerpo y subjetividad. Bogotá, Colombia: Editorial Universidad Central e Instituto para la Investigación Educativa y el Desarrollo Pedagógico (IDEP).

Chavarriaga, F y Ochoa, N. (2015). Efectos del disciplinamiento en la escuela en la configuración de subjetividad. (Tesis de Maestría). Universidad de Manizales. Colombia, Manizales.

Gallo, L. (2009). El cuerpo en la educación da qué pensar: perspectivas hacia una educación corporal. Estudios pedagógicos (Valdivia), 35(2), 232-242. Doi: https://dx.doi.org/10.4067/S0718-07052009000200013

Gómez, W. (2009). El cuerpo en la escuela: los dispositivos de sujetación. Curriculo sem Fronteiras. 9(1), 159-179.

Meza, V. y Rojas, I. (2017). Caracterización y evaluación de la comunicación no verbal en los estudiantes de $9^{\circ}$ en la clase de lengua castellana para mejorar sus prácticas expositivas por medio de un proyecto pedagógico de aula. (Tesis Maestría). Universidad de San Buenaventura. Cali, Colombia.

Muchemwa, S. (2013). Use of Nonverbal Communication in the Classroom as a Way of Enhancing Classroom Teaching. A Case Study of Solusi High School, Zimbabwe, Procedia - Social and Behavioral Sciences, 103(26), 1279-1287. http://www.sciencedirect.com/science/article/pii/S1877042813039025

Niño, C. (2015) "El cuerpo en la escuela subjetividades de jóvenes de las Instituciones Educativas Distritales Alemania Unificada y Fabio Lozano Simonelli de la ciudad de Bogotá D.C." (Tesis Maestría). Universidad Pedagógica Nacional, Bogota.

Pan, Q. (2014). "Nonverbal Teacher-student Communication in the Foreign Language Classroom" English Department, Zhenjiang Watercraft College, Zhenjiang, China. Theory and Practice in Language Studies, 4(12) 2627-2632. Recuperado de http://www.academypublication.com/issues/past/tpls/vol04/12/28.pdf

Paternain, C. (2016). El lenguaje corporal en el proceso de enseñanza-aprendizaje de la lengua extranjera: inglés. (Tesis de Maestría). Universidad de Valladolid. Valladolid, España. Recuperado de https://uvadoc.uva.es/bitstream/1 0324/20440/1/TFG-G\%202012.pdf

Pateti, Y. (2007). Reflexiones acerca de la corporeidad en la escuela: hacia la despedagogización del cuerpo. Paradigma, 28(1), 105-130. Recuperado de https://bit.ly/2mpEZnf

Pedraza, Z. (2010). Saber, Cuerpo y Escuela: el uso de los sentidos y la educación somática. Revista de investigación en el campo del arte, 4(5), 44-56.

Potayos, F. (2003). La comunicación no verbal: algunas de sus perspectivas de Estudio e Investigación. Revista de Investigación Lingüistica, 6(2), 67-83.

Rocha, A. (2000). El cuerpo como centro de interpretación: Una aproximación a la concepción nietzscheana. Universitas Philosophica, 17(34-35), 159-178. Recuperado de https://revistas.javeriana.edu.co/index.php/vnip hilosophica/article/view/11380

Scharagrodsky, P. (2007). El Cuerpo en la Escuela. Argentina: Ministerio de Educación, Ciencia y Tecnología. Recuperado de http://www.bnm.me.gov.ar/gigal/documentos/EL002216.pdf

\section{BY-NC-ND}

